\title{
Sustainability in biobanking
}

\author{
Maram Abdaljaleel ${ }^{1}$, Elyse J. Singer ${ }^{2}$, William H. Yong ${ }^{1,3,4,}{ }^{*}$ \\ ${ }^{1}$ Department of Pathology and Laboratory Medicine, David Geffen School of Medicine at UCLA, \\ Los Angeles, CA, 90095 \\ ${ }^{2}$ Department of Neurology, David Geffen School of Medicine at UCLA, Los Angeles, CA, 90095 \\ ${ }^{3}$ Brain Tumor Translational Resource, David Geffen School of Medicine at UCLA, Los Angeles, \\ CA, 90095 \\ ${ }^{4}$ Jonsson Comprehensive Cancer Center, David Geffen School of Medicine at UCLA, Los \\ Angeles, CA, 90024
}

\section{Summary}

i.

Biobanks are storage places for biospecimens that can be used for current and future scientific research. Biospecimens are exceptional sources of biological data that can be potentially translated from molecular and genetic information to clinically relevant treatment modalities. Examples of such biospecimens include, but are not limited to, blood, skin, hair, saliva, stem cells, DNA, and RNA. The volume of biospecimens worldwide continues to grow at an extraordinary rate posing a challenge for biobanks to manage this growth. Due to the vital role of biobanks in research, an understanding of biobanking sustainability is important. Simply starting to collect biospecimens without strategic planning and cost analysis can lead to failure. Components vital to sustainability include fostering public support, cost effective banking, funding development, standardized protocols, and interoperability.

\section{Keywords}

Biobank; Biospecimens; Sustainability; Interoperability; Standards; Accreditation

\section{Introduction}

The United Nations has estimated the world population to be 7.2 billion and projects it to reach 8.5 billion by 2030 (1). This growth in the world population will be paralleled by substantial biospecimen volume growth, providing a powerful scientific resource for academic, governmental, and pharmaceutical institutions worldwide $(2,3)$. Biobanks have the potential to foster technologies and scientific discoveries that provide greater understanding of disease processes, ultimately leading to treatments that can improve and help achieve optimal patient health. Understanding sustainability is required in order to

"Corresponding author: William H. Yong M.D., Brain Tumor Translational Resource, David Geffen School of Medicine at UCLA, CHS 13-145B, 10833 Le Conte Avenue, Los Angeles, CA, 90095 USA. Ph: (310) 825-8269 FAX: 310-825-7353;

WYong@mednet.ucla.edu. 
provide a solid foundation for future biorepositories. Some biobanks have closed or were acquired by other entities. The Singapore Bio-Bank (SBB), which was established in 2002, is an example of a biorepository that closed in part due to a lack of self-sufficiency, a perceived high cost of resource access by researchers, low utilization of biospecimens, and a loss of public and governmental support (3). It is a common challenge for biobanks that the true cost of a biospecimen is prohibitive for researchers and that subsidization by the home institution, charity or governmental agencies is necessary. In this chapter, we will discuss issues in sustainability and some of the elements that can help a biobank thrive and successfully support scientific research.

\section{Sustainability through targeted and cost-effective biobanking}

Sustainability can be defined as "the endurance of systems and processes". In terms of biobanking, sustainability can be considered within a frame of three main factors: financial, operational, and social, as proposed by Peter Watson et al. (4). The ability of a biorepository to secure stable sources of funding, ability to standardize procedures and protocols that guarantee the highest quality tissue, and compliance with legal, social, and privacy regulations, are all important factors that will determine its endurance.

Traditionally, biospecimens have been collected and procured with minimal thought to the goals, cost, and required infrastructure, due to which the cost of sustaining biobanks is often underestimated. Financial considerations are not limited to the price of a freezer or two. There are costs of personnel needed to procure, store, and dispense the specimens, costs related to informatics requirements such as software and hardware, and costs related to maintaining and monitoring freezers-electricity, carbon dioxide or liquid nitrogen back-up, remote monitoring, repairs, and freezer replacement after 8-10 years. As banks of freezers can generate substantial heat, there is a need of temperature-controlled rooms as well. The running costs of biobanks are dependent on the duration of the project, number of specimens, and the purpose of storing them. The need to store more specimens correlates directly with the world's growing population, increased access to healthcare, and advancements in technology that can produce increasingly sophisticated characterization of biospecimens. The duration over which specimens need to be sustained before they provide significant value is quite variable, which requires that the cost effectiveness of the process should be thought through carefully. The number of specimens needed for a particular study will depend on its statistical power requirements. Population-based epidemiological cohort studies may require a large number of biospecimens that need to be stored for long periods of time. Consideration should be given to how biospecimens are to be stored. If such specimens can be stored at room temperature rather than in ultra-cold conditions, the storage costs diminish substantially. Given the projected exponential growth of patients and potential biospecimens, it is not feasible to collect everything and to store them forever. Weighing the sustainability costs of each project in terms of duration and project outcome is important, and costs should be delineated prior to the start of biospecimen procurement with the expected benefits and potential impact on scientific outcome or human health clearly defined. This fiscal responsibility is necessary to encourage public and private entities to invest in and to fund biorepositories. 


\section{Sustainability through standardization and accreditation}

Operationally, biobanking sustainability is strengthened by the presence of standardized protocols and procedures that assure quality control in all aspects of the biobanking process. These standardized protocols help to achieve high quality biospecimens that lead to end-user research community satisfaction and meaningful research that rewards the patient's generosity and trust in donating their biospecimens. Standard operating procedures may encompass donor consent, tissue quality assurance, type of project, adequate personnel and facilities, and linked de-deidentified clinical data. Obstacles to sustainability also include social, legal, ethical, and privacy-related issues. The variation in laws between countries, along with the diverse social views of various aspects of the biobanking process is a challenge in procurement itself and in facilitating collaboration between biobanks. If appropriately designed, the standard operating procedures can help maintain compliance with local and international laws, rules and regulations. Abrogation of laws can result in the closure of a biobank or potentially suspension of governmental funding of an entire institution. Ethical and legal issues are present in many aspects of biobanking, with the most prominent concerns being informed consent, privacy protection, ownership of intellectual property, participation of minors and incompetent individuals, and returning results to participants $(5,6,8)$. Adherence to established consenting protocols and transparency can provide reassurance to patients, their families and the public. Patients want to help advance research that can produce cures for themselves and, if not for themselves, for their families and for others. Treating them fairly and considerately is part and parcel of repaying the patients and also earning the support of patient advocacy groups that can be powerful supporters of the biobank.

Biobank accreditation consists of objective testing and observation that assures the standardization of protocols and procedures. The biorepository accreditation program instituted by the College of American Pathologists (CAP) assesses compliance with standards through on-site inspections occurring every three years. The CAP accreditation program is available to biobanks in America and Canada and is not applicable for tissue stored for transplant purposes. There is a moderate but not insignificant cost involved and accreditation may not be affordable for small biobanks. Similar inspection processes are available in other countries. It is anticipated that biobank accreditation will eventually be a requirement of major national funding agencies.

\section{Sustainability through collaboration, education and advocacy}

Another important step to enhancing sustainability is working closely with and educating the public, funding institutions, researchers, and biomedical thought leaders of the importance and value of biobanks. If a biobank is sited in a university or medical center, close collaboration with end-users to include funding requests for biospecimen procurement in their grant proposals is a must. Initial low cost or pro bono collaborations to gather preliminary data can lead to successful grant funding for both the biobank and the researcher in the long run. A biobank that successfully yields publications and research grants provides concrete evidence of its inherent value. Advocacy by patients, patient advocacy groups, oncology and surgical colleagues, and the local cancer center can drive strategic initiatives 
by one's own institution to support the biobank. Workshops and conferences aimed at discussing the positive economic and social impact of biorepositories are of value. Advocacy by patients as well as medical or scientific associations has the potential to modify government policies in regards to tax benefits and increased funding for biorepositories. Developing collaborations between biorepositories and pharmaceutical companies is also a consideration. Pharmaceutical companies help to drive the development and use of novel therapeutics: they have an important and constructive role to play in society. Although industry relationships can support biomedical advances, the perceived commercialization of specimens is controversial from an ethical standpoint $(7,8,9)$. Patients tend to trust academic research studies more than industry studies. So, collaborations should be approached with clear sightedness, and institutional safeguards, perhaps fortified with patient advocate input, should be in place to protect the public good.

\section{Sustainability through interoperability}

Interoperability means that specimens and their scientifically relevant data have been collected, processed, and archived within an ethical and legal framework, that provides those conducting the research a way to easily combine them with related items from other biorepositories (10). It is an essential component of fostering national and international multi-center research collaborations. Interoperability provides the best usage of biospecimens and their relevant data by potentially involving the same tissue in multiple projects or more likely providing statistical power for a study by leveraging many specimens from multiple biorepositories. Funding agencies are very interested in synergistic collaborations and a clear demonstration of interoperability can enhance a grant application. Although interoperability has clear benefits, legal and ethical concerns must be overcome. Ownership of specimens and data, privacy, and utilization of specimens by more than one biorepository, are some of the thorny issues that confront interoperability (11). Simplification and harmonization of national laws can facilitate international collaborations. Unification of patient advocacy groups

The until recent absence of national and international standards and their nascent use in different countries are other obstacles to interoperability. Creating and using standards that are nationally and internationally agreed upon is an important step to overcoming these road blocks. The International Society for Biological and Environmental Repositories (ISBER) has made great strides in that direction and their online resources, particularly their Recommended Best Practice for Biorepositories, are a must read for biobankers. While one typically thinks of biobanks as rooms with freezers or as collections of physical biospecimens, the "virtual" biorepository wherein the metadata that represents these biospecimens and their associated clinical and molecular data resides is the key to identifying required biospecimens with the desired characteristics for any given multi-center study. The ability for each biobank's data repository or information system to talk to each other is critical for interoperability. The usage of Common Data Elements (CDEs) and controlled vocabularies are recommended by both the Office of Biorepositories and Biospecimen Research (OBBR) (12) and the International Society for Biological and Environmental Repositories (ISBER) (13). Controlled vocabulary is a restricted list of words or terms used for labeling, indexing, or categorizing, and is used as a means to standardize 
information for the purposes of capturing, storing, exchanging, searching, and analyzing data (14). It is controlled because only vocabulary from the list can be used for the subject area covered by the controlled vocabulary. Examples of such standardized vocabulary created for healthcare are the Systematized Nomenclature of Medicine Clinical Terms (SNOMED-CT), Logical Observation Identifiers Names and Codes (LOINC), and the Unified Medical Language System (UMLS) (15). Common Data Element (CDE) is a data element that is common to multiple data sets across different studies, and is used to help identify, compare, and combine multiple studies (16). As more information systems use common vocabularies and data elements, interoperability will be enhanced creating greater value in a biobank and ultimately promoting sustainability.

\section{Conclusion}

Sustaining a biobank is costly but its value is incalculable as biospecimens are critical substrates for biomedical research. The biobanking community has made good strides forward but major challenges remain to be overcome in order to improve the sustainability of biobanks. It is only by working with patients, researchers, governmental entities and other stakeholders that biobanks can thrive and even expand the vital biospecimen pipeline.

\section{Acknowledgement}

This work was supported in part by NIH:NCI P50-CA211015, NIH:NIMH U24 MH100929, the Art of the Brain Foundation, and the Henry E. Singleton Brain Cancer Research Program.

\section{References}

1. UN projects world population to reach 8.5 billion by 2030 , driven by growth in developing countries | UN News. (n.d.). Retrieved from https://news.un.org/en/story/2015/07/505352-un-projects-worldpopulation-reach-85-billion-2030-driven-growth-developing

2. McDonald Sandra A. et al. (2012) "Fee-for-Service as a Business Model of Growing Importance: The Academic Biobank Experience," Biopreservation and Biobanking 10, no. 5: 421-25, doi: 10.1089/bio.2012.0017.

3. Chan Tuck Wei, The Closure of the National Bio-bank in Singapore, http://www.asiabiotech.com/ publication/apbn/16/english/preserved-docs/1604/0040_0043.pdf. accessed October 21, 2015,

4. Watson Peter H. et al. (2014) "A Framework for Biobank Sustainability," Biopreservation and Biobanking 12, no. 1: 60-68, doi:10.1089/bio.2013.0064.

5. Hansson Mats G. (2011) "The Need to Downregulate: A Minimal Ethical Framework for Biobank Research,” Methods in Molecular Biology (Clifton, N.J.) 675: 39-59, doi: 10.1007/978-1-59745-423-0_2.

6. Helgesson Gert et al. (2007) "Ethical Framework for Previously Collected Biobank Samples," Nature Biotechnology 25, no. 9: 973-76, doi:10.1038/nbt0907-973b.

7. Rothstein Mark A. (2005) "Expanding the Ethical Analysis of Biobanks," The Journal of Law, Medicine \& Ethics: A Journal of the American Society of Law, Medicine \& Ethics 33, no. 1: 89101.

8. Hawkins Alice K. (2010) "Biobanks: Importance, Implications and Opportunities for Genetic Counselors,” Journal of Genetic Counseling 19, no. 5: 423-29, doi:10.1007/s10897-010-9305-1.

9. Joly Yann and Knoppers Bartha M. (2006) "Pharmacogenomic Data Sample Collection and Storage: Ethical Issues and Policy Approaches," Pharmacogenomics 7, no. 2: 219-26, doi: 10.2217/14622416.7.2.219.

10. Kiehntopf M, Krawczak M, (2011), Biobanking and International Interoperability: Samples Springer, doi:10.1007/s00439-011-1068-8. 
11. Hawkins Alice K and O’Doherty Kieran C (2011) “'Who Owns Your Poop?': Insights Regarding the Intersection of Human Microbiome Research and the ELSI Aspects of Biobanking and Related Studies,” BMC Medical Genomics 4: 72, doi:10.1186/1755-8794-4-72. [PubMed: 21982589]

12. Welcome to the Biorepositories and Biospecimen Research Branch (BBRB), http:// biospecimens.cancer.gov/default.asp. accessed October 19, 2015

13. ISBER, http://www.isber.org/. accessed October 19, 2015

14. BIOBANKING; Advancing Biorepositories with Data Science (2014) http://cdn2.hubspot.net/hub/ 111084/file-510108713-pdf/docs/5AM_Solutions_eBook-Biobanking-

Advancing_Biorepositories_with_Data_Science.pdf., accessed October 21, 2015,

15. Supporting Interoperability - Terminology, Subsets and Other Resources from NLM. https:// www.nlm.nih.gov/hit_interoperability.html Accessed 1 Oct 2018

16. Glossary, FAQs, Help Files, Pocket Cards, https://www.nlm.nih.gov/cde/ glossary.html\#cdedefinition, accessed October 21, 2015, 